\title{
Longitudinal neuroimaging correlates of subjective memory impairment: 4-year prospective community study
}

\author{
Robert Stewart, Ophélia Godin, Fabrice Crivello, Pauline Maillard, Bernard Mazoyer, \\ Christophe Tzourio and Carole Dufouil
}

\section{Background}

complaints about memory are common in older people but their relationship with underlying brain changes is controversial.

\section{Aims}

To investigate the relationship between subjective memory impairment and previous or subsequent changes in white matter lesions and brain volumes.

\section{Method}

In a community cohort study of 1336 people without dementia, 4-year changes in brain magnetic resonance imaging measures were investigated as correlates of subjective memory impairment at baseline and follow-up.

\section{Results}

Subjective memory impairment at baseline was associated with subsequent change in hippocampal volume and at follow-up impairment was associated with previous change in hippocampal, cerebrospinal fluid and grey matter volume and with subcortical white matter lesion increases. All associations with volume changes were U-shaped with significant quadratic terms - associations between least decline and subjective memory impairment were potentially explained by lower baseline hippocampal volumes in the groups with least volume change. Associations between hippocampal volume change and subjective memory impairment at follow-up were independent of cognitive decline and depressive symptoms, they were stronger in participants with the apolipoprotein E (APOE) $\varepsilon 4$ allele and in those without baseline subjective memory impairment.

\section{Conclusions}

Complaints of poor memory by older people, particularly when new, may be a realistic subjective appraisal of recent brain changes independent of observed cognitive decline.

\section{Declaration of interest}

\section{None.}

Subjective memory impairment is one of few potential presenting symptoms for people with early cognitive impairment and has been a core feature of many criteria for mild cognitive impairment or equivalent constructs. ${ }^{1}$ However, its 'validity' remains controversial. For example, several longitudinal studies have found that dementia incidence is higher in people who admit to memory difficulties, ${ }^{2-4}$ but the positive predictive value is low. ${ }^{5}$ Although associations with contemporaneous cognitive function or subsequent decline have been variable, ${ }^{6-8}$ those between subjective memory impairment and hidden risk factors for dementia have been more consistently found and include the presence of the apolipoprotein E (APOE) $\varepsilon 4$ allele, ${ }^{9,10}$ Alzheimer neuropathology, ${ }^{11}$ white matter lesions, ${ }^{12-14}$ lower contemporaneous cerebral volume generally ${ }^{15,16}$ and lower hippocampal volume specifically. ${ }^{14,17}$ To our knowledge, there has been no previous prospective neuroimaging research in relation to subjective memory impairment. Using data from a 4-year prospective community study of older people with neuroimaging repeated over this period, our objectives were to investigate associations between subjective memory impairment and both subsequent and previous changes in brain volumetric measures and white matter lesions, hypothesising that magnetic resonance imaging (MRI) changes indicative of worsening brain structure would be associated with subjective memory impairment both at baseline (i.e. subjective memory impairment predicting future brain changes) and at follow-up (i.e. subjective memory impairment reflecting previous brain changes). Magnetic resonance imaging changes of interest in this respect were white matter, grey matter and cerebrospinal fluid (CSF) volumes, hippocampal volumes and white matter lesion volumes (periventricular and subcortical).

\section{Method}

\section{The baseline sample}

The baseline sample and measurements have been described previously. ${ }^{14}$ In brief, the $3 \mathrm{C}$ Study is a multicentre cohort study carried out in three French cities (Bordeaux, Dijon and Montpellier) of community-dwelling adults aged 65 and over. ${ }^{18}$ The study protocol was approved by the Ethical Committee of the University Hospital of Kremlin-Bicêtre, and participants signed informed consent. A cerebral MRI substudy was performed among participants in Dijon. Usual MRI exclusion criteria were applied. Of 2763 participants aged less than 80 approached for MRI, 2285 agreed to participate. As a result of financial limitations, 1924 examinations were carried out. Compared with participants who did not have MRI $(n=839)$, those who did were significantly younger (mean age $72.5($ s.d. $=4.1)$ v. $73.4($ s.d. $=4.0)$ years, $P<0.001)$, were less likely to be female $(62.2 \%$ v. $71.0 \%$, $P<0.001)$, more often had an education level above baccalaureate (23.5\% v. $17.8 \%, P<0.001)$ and were more likely to report good or very good health $(62.3 \%$ v. $56.4 \%, P<0.001)$. Participants with poor-quality scans $(n=123)$ or dementia at baseline $(n=8)$ were excluded.

\section{Follow-up}

All surviving participants were invited for re-examination 2 and 4 years after inception. Subjective memory impairment was ascertained in an identical way (see below) at all interviews. The MRI measures were estimated at the 4-year follow-up, using identical procedures for acquisition and analysis to those used at 
baseline. ${ }^{19}$ For the analyses presented here only baseline and 4-year measures were considered.

\section{MRI acquisition and analysis}

Magnetic resonance imaging acquisition was performed on a 1.5 Tesla Magnetom (Siemens, Erlangen) at both baseline and follow-up using identical protocols. Fully automated procedures were used to ascertain both volumetric and white matter lesions data, which have been described in detail previously, ${ }^{14,20,21}$ and which were also identical for images taken at baseline and follow-up. For this analysis, total, periventricular and subcortical white matter lesion volumes were calculated and expressed as a proportion of white matter detection mask. The following volumetric data were used: grey matter, white matter, CSF and hippocampal volumes. For all MRI measures, baseline values were subtracted from follow-up values in order to calculate change scores.

\section{Subjective memory deficit}

In a series of questions about perceived difficulties, all participants were asked at baseline and 4-year follow-up (in identical questionnaires) whether they had habitual forgetfulness during daily activities and whether they had difficulties remembering recent new information. Although these were not defined from a specific scale, they were chosen on the basis of previous research in French community samples using similar brief measures. ${ }^{22}$ Participants who responded positively to both these questions were defined as having subjective memory impairment and were compared with the remainder of the sample at the respective examination for all analyses, an approach which defined a similar prevalence to those reported for problematic subjective memory impairment in other community settings. ${ }^{9,23}$

\section{Other measures}

The following were ascertained at baseline: age, gender, level of education (seven categories of duration of formal education) and presence or absence of APOE $\varepsilon 4$. Additional covariates ascertained over the follow-up period included: incident stroke, incident dementia and depressive symptoms at follow-up (Center for Epidemiological Studies Depression Scale ${ }^{24}$ (CES-D)). Dementia diagnoses were based on neuropsychological testing, medical history, neurologist review and consensus review, applying standard criteria. ${ }^{25}$ Subtypes were not analysed here but have been reported previously for the $3 \mathrm{C}$ samples. ${ }^{26}$ Suspected stroke occurrence was confirmed by medical records where possible and an end-point adjudication committee. Change (follow-up minus baseline) scores were calculated for the following cognitive tests that had been administered in an identical manner at baseline and follow-up: Mini-Mental State Examination (MMSE); ${ }^{27}$ Isaac's Set Test (testing global verbal fluency); ${ }^{28}$ Benton Visual Retention Test (testing recall of previously presented designs). ${ }^{29}$

\section{Statistical analysis}

All data were analysed using STATA 10 software for Windows. The sample was restricted to participants with complete MRI data at both baseline and year 4. Change scores for MRI measures were divided by quintiles into five equal groups and considered as independent variables in regression models. For primary analyses, subjective memory impairment frequency was compared across the groups, hypothesising linear (monotonic) associations. Two sets of analyses were carried out: the first set modelled subjective memory impairment at baseline as a binary dependent variable against MRI changes (i.e. investigating associations between subjective memory impairment and subsequent brain change); the second set modelled subjective memory impairment at 4-year follow-up against MRI changes (i.e. investigating associations between subjective memory impairment and previous brain change). Logistic regression models were used to investigate associations further. Where curvilinear associations were suggested, quadratic terms were fitted and tested for independent neuroimaging variables (quintiled scores). The following covariates were entered sequentially: age, gender, education and incident stroke. Further separate adjustments were then made for change in cognitive function, incident dementia and year-4 CES-D score. Finally, exploratory stratification was carried out for presence or absence of APOE $\varepsilon 4$; MMSE change (stable/ improving $v$. decline); MMSE level at the time of the subjective memory impairment measure; and presence or absence of subjective memory impairment at the other time point.

\section{Results}

Of 1792 participants without dementia and with MRI data at baseline, $1337(74.5 \%)$ participated in the MRI study at followup (50 had died, 130 were lost to follow-up and the remaining 275 were not offered a follow-up MRI because of financial constraints). Compared with the total baseline sample, those followed were slightly younger, more likely to be female and less likely to have lower education (Table 1). They did not differ substantially in terms of MMSE score or subjective memory impairment at baseline. Characteristics of the sample at 4-year follow-up are also displayed in Table 1. Subjective memory impairment was reported at baseline by $26.6 \%$, at follow-up by $26.0 \%$ and on both occasions by $10.3 \%$.

Associations between MRI volume changes over the 4-year follow-up period and subjective memory impairment at the beginning of that period are summarised in Table 2. Because some associations appeared non-linear (U-shaped), the respective quadratic term was also tested for each quintiled independent variable. This was significant for hippocampal volume change and bordered on statistical significance for CSF volume change. The association between baseline subjective memory impairment

Table 1 Sample characteristics at baseline and follow-up

\begin{tabular}{|lcc|}
\hline & $\begin{array}{c}\text { Baseline } \\
(n=1793)\end{array}$ & $\begin{array}{c}\text { Follow-up } \\
(n=1336)\end{array}$ \\
\hline Age at baseline, mean (s.d.) & $72.4(4.1)$ & $72.0(4.0)$ \\
\hline Gender: female, \% & 60.4 & 61.8 \\
\hline Less than secondary education, \% & 16.4 & 15.4 \\
\hline Baseline MMSE, mean (s.d.) & $27.6(1.8)$ & $27.8(1.7)$ \\
\hline Baseline CES-D, mean (s.d.) & $9.1(7.9)$ & $8.7(7.6)$ \\
\hline APOE genotype: $\varepsilon 4$ present, \% & 22.5 & 21.9 \\
\hline $\begin{array}{l}\text { Subjective memory impairment } \\
\text { at baseline, \% }\end{array}$ & 26.6 & 26.0 \\
\hline $\begin{array}{l}\text { Subjective memory impairment } \\
\text { at year 4, \% }\end{array}$ & - & 20.1 \\
\hline Incident stroke since baseline (\%) & - & 0.8 \\
\hline Incident dementia since baseline (\%) & - & 0.5 \\
\hline MMSE at year 4, mean (s.d.) & - & $-0.1(1.8)$ \\
\hline Prior change in MMSE, mean (s.d.) & - & $9.0(7.9)$ \\
\hline CES-D at year 4, mean (s.d.) & - & \\
\hline $\begin{array}{l}\text { MMSE, Mini-Mental State Examination; CES-D, Center for Epidemiological Studies } \\
\text { Depression Scale; APOE, apolipoprotein E. }\end{array}$ & \\
\hline
\end{tabular}




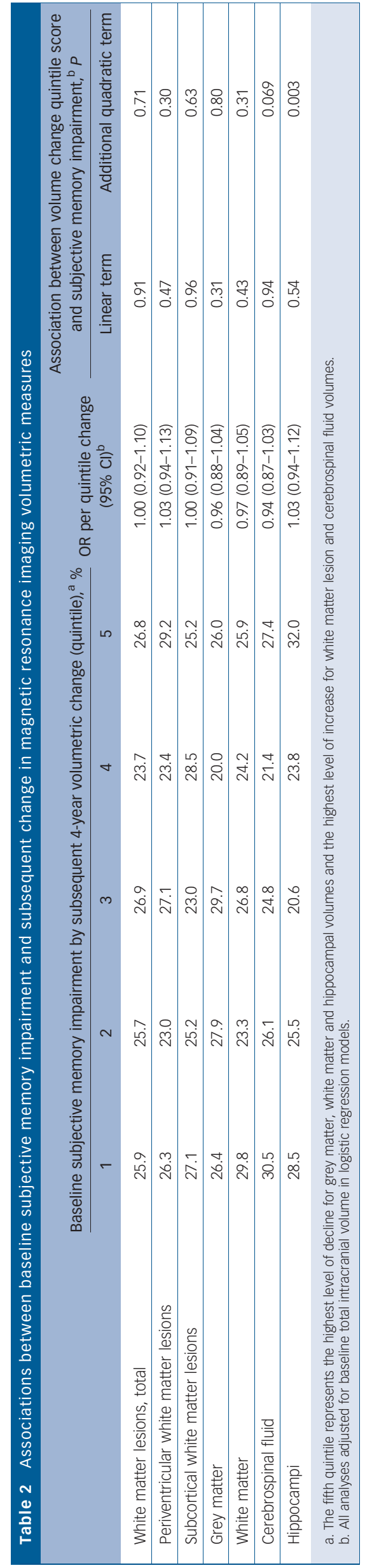

and subsequent hippocampal volume change was investigated in further logistic regression models and remained significant after adjustment for age, gender, education, incident stroke, baseline MMSE score and baseline CES-D score (adjusted odds ratios (ORs) for linear and quadratic terms in the same model: 0.53 $(P=0.006)$, and $1.11(P=0.005)$ respectively $)$.

Associations between MRI volume changes over the 4-year follow-up period and subjective memory impairment prevalence at the end of that period are summarised in Table 3. Quadratic models implying U-shaped associations were significant for grey matter, CSF and hippocampal volume change and subcortical white matter lesion volume change. These were investigated in further logistic regression models, summarised in Table 4. All associations were robust to adjustment for age, gender, education, incident stroke, year-4 CES-D score and change scores for all three repeated cognitive tests, the coefficients showing little change after any adjustment. Although some $P$-values fell below significance levels, there were not substantial changes in the coefficients when the four volume change measures (both linear and quadratic terms) were entered simultaneously into the same model and the sizes of respective coefficients were similar.

In stratified analyses of the association between subjective memory impairment at follow-up and previous volume change (Table 4), coefficients for all four MRI measures were stronger in participants with the APOE $\varepsilon 4$ allele but showed little consistent or substantial modification by MMSE change or MMSE score at follow-up. Associations between subjective memory impairment and prior CSF or hippocampal volume changes were stronger if subjective memory impairment had not been present at baseline - i.e. were stronger for new-onset subjective memory impairment over the follow-up period.

A further secondary analysis was carried out to clarify potential reasons for the U-shaped associations, in particular the relationship between brain volume at baseline and subsequent change in volume, given previous findings of strong associations between lower hippocampal volume and subjective memory impairment at baseline in this cohort. ${ }^{14}$ For hippocampal measures, baseline mean volumes $\left(\mathrm{cm}^{3}\right)$ by quintiled change score (ordered from least to most volume loss as defined for Tables 2 and 3) were $6.79,6.84,6.70,6.67$ and 6.37 respectively - an inverted U-shaped distribution that indicated least hippocampal volume loss in those with relatively small hippocampi at baseline. A linear regression of the association between change in hippocampal volume (dependent variable) and baseline hippocampal volume (independent variable) gave similar and significant findings (linear coefficient $0.25, P=0.004$; quadratic coefficient $-0.02, P=0.009)$. The same patterns were observed in baseline mean hippocampal volumes ordered by quintiled change scores representing increasing CSF volume (6.59, 6.62, $6.76,6.77$ and 6.64 respectively) and decreasing grey matter volume $(6.64,6.69,6.70,6.71$ and 6.64 respectively). However, an alternative model, entering quintiled scores of hippocampal volume change expressed as a proportion of baseline hippocampal volume gave similar U-shaped associations for year-4 subjective memory impairment as a dependent variable (after adjustment for age, gender, education and stroke: linear term for descending quintile groups $\mathrm{OR}=0.62 \quad(95 \%$ CI $0.44-0.87, \quad P=0.006)$; quadratic term $\mathrm{OR}=1.16$ (95\% CI 1.07-1.25, $P<0.001)$.

\section{Discussion}

\section{Main findings}

In a large community sample with repeated neuroimaging data obtained over a 4-year period, we investigated associations 

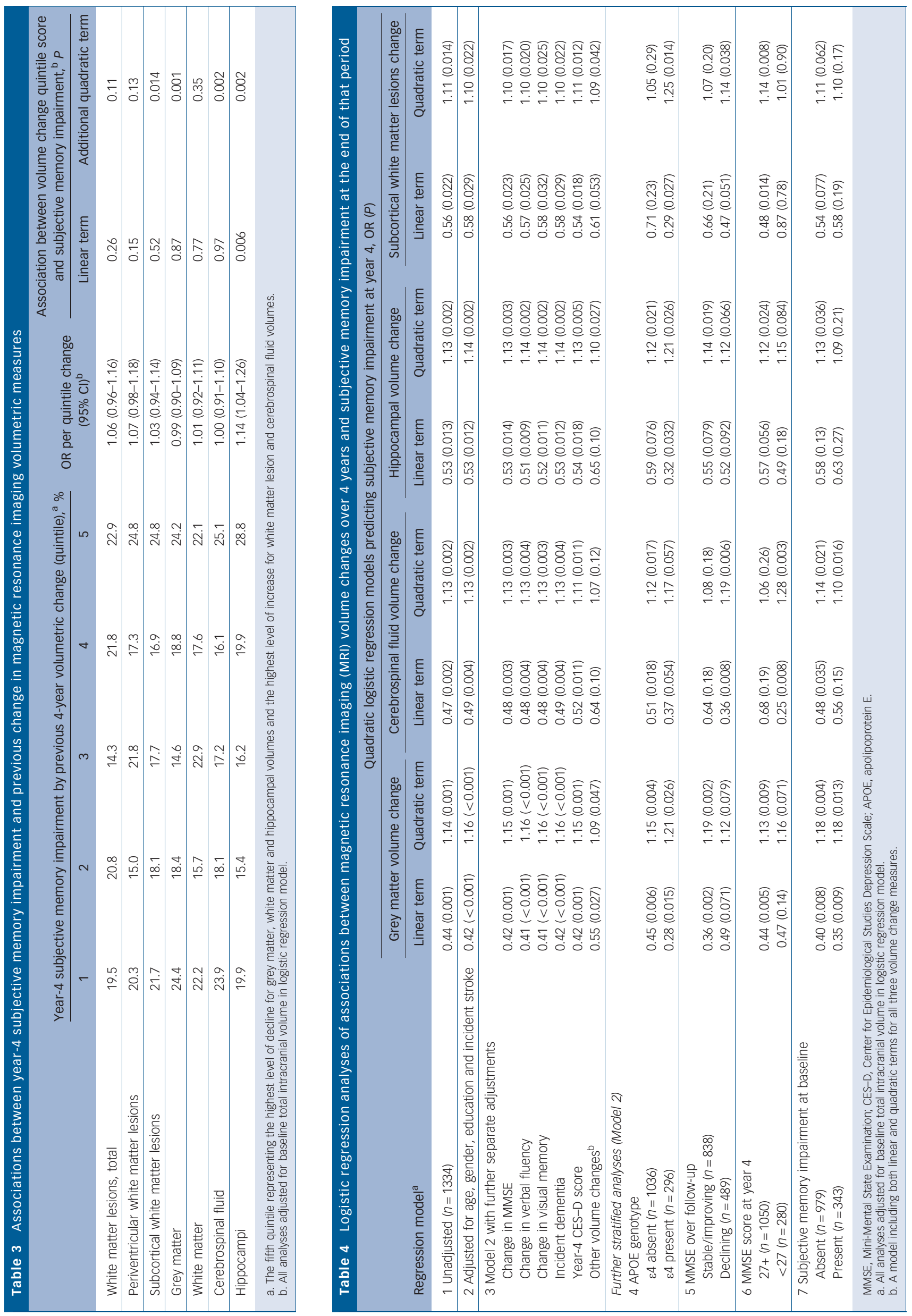
between changes in volumetric measures/white matter lesions and subjective memory impairment at baseline and follow-up. The first set of analyses investigated associations between baseline subjective memory impairment and subsequent MRI change, whereas the second set investigated associations between follow-up subjective memory impairment and preceding MRI change. We found stronger evidence for associations with preceding rather than subsequent volume changes: specifically for grey matter, CSF and hippocampi as well as for subcortical white matter lesions. However, there was also a significant association between baseline subjective memory impairment and subsequent hippocampal volume change. All significant associations were U-shaped (discussed further below). For associations between follow-up subjective memory impairment and previous volume change, there was no evidence of confounding by other co-occurring events such as cognitive decline, dementia or depressive symptoms, and the different volume change measures appeared independent of each other as predictors. However, there was some evidence for modification by APOE genotype, and the associations were strongest for follow-up subjective memory impairment that was incident over the follow-up period (i.e. not present at baseline).

\section{Strengths and limitations}

Strengths of the study included a large, well-characterised community sample and a reasonable rate of follow-up. The analyses tested a priori hypotheses using the data available from this valuable cohort, although the study as a whole was not set up to address these. Neuroimaging data, obtained using an automated technique, are unlikely to be biased with respect to subjective memory impairment, although it should be borne in mind that the subsample in whom MRI measures were available was not completely representative of the source population. However, although there was attrition bias by age and education, the strength and pattern of association between subjective memory impairment and neuroimaging measures are less likely to be influenced in this respect. Subjective memory impairment itself was defined from a relatively brief measure comprising responses to two questions. This approach has been commonly adopted in studies of subjective memory impairment, an advantage being that it is more readily generalisable than a scale measure to clinical and other community settings (as the questions are easily asked). Its disadvantage is that brief instruments are more subject to measurement error or reduced test-retest reliability. However, misclassification of this nature will obscure rather than exaggerate true associations and suboptimal measurement will not account for the identified significant associations. 'Validity' is a complex construct when applied to subjective memory impairment measures. On one level, a complaint of poor memory, however briefly assessed, has to be taken as genuine at the time it is ascertained. A more popular approach to validity however has been to evaluate the extent to which subjective memory impairment is indicative of underlying brain function or pathology, an issue which will be considered below.

\section{The validity of subjective memory impairment in older people}

Subjective memory impairment is an important symptom because it is one of a few ways in which people with early neurodegeneration might present to clinical services. Early studies had suggested that complaint about poor memory by an older person was more indicative of depression than early dementia. ${ }^{30}$ This may still be the case since associations with depression are invariably strong in community samples. However, the traditional view of subjective memory impairment as a symptom poorly reflective of brain function has been challenged by more recent research, in particular research that has found associations with an increased risk of subsequent dementia. ${ }^{2-5}$ The positive predictive value in this respect is relatively low, ${ }^{5}$ but this may simply reflect the low incidence of dementia in community samples and modifying factors. For example, associations between subjective and objective cognitive function have been found to be stronger in more cognitively demanding environments. ${ }^{23,31}$ Furthermore, the likelihood of subjective memory impairment being reported in people with preclinical dementia may well depend on the extent and pattern of underlying neurodegeneration since loss of insight (i.e. 'disappearance' of subjective memory impairment ) appears to occur close to the clinical onset of dementia in at least some cases. ${ }^{3}$ Some people with early dementia may therefore not notice memory deficits because these are not interfering with daily tasks and, by the time they do, insight may have been lost.

Further evidence for the 'validity' of subjective memory impairment has come from studies that have investigated contemporaneous associations with proxy markers of brain pathology or dementia risk. Two studies have found associations between subjective memory impairment and APOE $\varepsilon 4,9,10$ although others have not. ${ }^{3,32}$ More consistent associations have been reported with white matter lesions, ${ }^{12,13}$ lower cerebral volume $e^{15,16}$ and, specifically, smaller hippocampal volume in a sample with white matter lesions. ${ }^{17}$ One study also found associations between ante-mortem subjective memory impairment and post-mortem Alzheimer neuropathology. ${ }^{11}$ In a cross-sectional analysis carried out at baseline in the cohort described here, we found associations between subjective memory impairment and both higher white matter lesion volume and smaller hippocampal volume, independent of depressive symptoms. ${ }^{14}$

To our knowledge, the prospective interrelationship between subjective memory impairment and MRI changes has not been investigated previously. In general, our findings in this analysis support the 'validity' of subjective memory impairment in that it was more frequently reported by participants with the highest levels of volume loss, whether this was measured as total grey matter or hippocampal volume loss or as CSF volume increase. No associations were found for changes in total or periventricular white matter lesion volume but an association was found between subjective memory impairment at follow-up and previous change in subcortical white matter lesion volume. Although the pattern of association with subjective memory impairment (U-shaped and with subsequent rather than previous subjective memory impairment) was similar to that of other volumetric change measures, it is less consistent with baseline cross-sectional associations with subjective memory impairment, which were stronger for periventricular lesions. ${ }^{14}$ Furthermore, some research suggests that subcortical lesions are more strongly associated with depression than dementia. ${ }^{33}$ This could possibly account for the association observed in this sample. However, adjustment for depressive symptoms (CES-D score) had little impact (Table 4). The lack of prospective associations with periventricular lesions may be explained by insufficient change at the higher end of the distribution over the 4 years of observation.

Associations between subjective memory impairment and volume changes, when present, were U-shaped with higher prevalence of subjective memory impairment in both those with most volume loss and those with least loss. We believe that low volumes at baseline being less likely to decrease at follow-up is the most likely explanation for the increased prevalence of 
subjective memory impairment in quintile one responsible for significant quadratic terms in Tables 2 and 3. Of all volumetric measures at baseline, hippocampal volume had been found to be the most strongly and robustly associated with subjective memory impairment in cross-sectional analyses. ${ }^{14}$ In a post hoc analysis we found that those with least decline on all three volume measures of interest had relatively low hippocampal volumes at baseline. This may define a group who had stable low volumes over the follow-up period and higher subjective memory impairment because of this. We have previously suggested that lower hippocampal volume associated with subjective memory impairment might reflect either hippocampal atrophy secondary to neurodegeneration or hippocampal volume attained or acquired at a much earlier stage. ${ }^{14}$ Our prospective findings suggest that both these explanations might be true - the quintile one group in Tables 2 and 3 representing stable low volume and the quintile five group representing declining volume. Whether stable low hippocampal volume in this group reflects the volume attained during early brain development or discrete loss of volume acquired later in life remains to be determined. This effect of baseline volume would have obscured associations between subjective memory impairment and 'low and declining' hippocampal volume and therefore does not explain the increased subjective memory impairment in quintile five groups in Tables 2 and 3.

\section{Clinical importance of subjective memory impairment}

How seriously then should a complaint of poor memory be taken in a clinical encounter? In general, associations between subjective and objective cognitive function have been absent or heterogeneous in previous research, ${ }^{6}$ which historically has led to an underestimation of the symptom's importance. However, interpretation of cognitive performance on a single occasion relies strongly on an individual's deviation from a demographic norm and that person's previous level of function can only in practice be estimated approximately from crystallised IQ measures. Furthermore, tests that are repeated over time may have insufficient accuracy to distinguish meaningful decline from random fluctuation and practice effects. In our sample, associations between subjective memory impairment and volumetric changes were little changed following adjustment for or stratification by change in or level of cognitive function, suggesting that subjective memory impairment may represent an appraisal of underlying function that is more accurate at least than the relatively brief assessments that are most commonly administered in clinical settings. They were also unchanged following adjustment for depressive symptoms. In general we found that subjective memory impairment reflected previous volume change more strongly than it predicted subsequent change, which is not surprising since there are likely to be a number of other determinants of subsequent change that may or may not be present at the time a memory deficit is noticed. A predictive association was found between subjective memory impairment and subsequent hippocampal volume change but the two-visit study design did not allow us to adjust for previous hippocampal volume change as a potential confounding factor.

Interestingly, associations with previous volume changes were strongest for subjective memory impairment that was present at follow-up but not at baseline - i.e. for 'incident' rather than 'persistent' subjective memory impairment. One previous prospective study found that people with incident dementia over a 2.5 year follow-up period were more likely to have 'persistent' or 'transient' subjective memory impairment for the same two examination points - i.e. subjective memory impairment that was present at both baseline and follow-up or subjective memory impairment that was present at baseline but not at follow-up (the second of these possibly reflecting loss of insight). ${ }^{3}$ In that study there was no association between dementia and 'incident' subjective memory impairment, implying that the onset must have been at an earlier stage of cognitive decline. Our findings support this since they were present in a relatively healthy sample very few of whom developed dementia and, for hippocampal volume and grey matter volume change, remained significant in those with MMSE scores of 27-30 at the end of the follow-up period and in those with no change in MMSE score over 4 years. They therefore suggest that subjective memory impairment, if it does manifest, does so at relatively mild levels of neurodegeneration before there is any evidence of cognitive impairment or decline, at least on brief assessments.

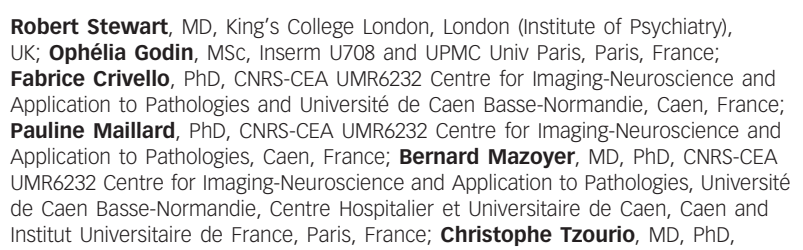

Correspondence: Carole Dufouil, INSERM Unit 708, Hôpital La Salpêtrière, 75651 Paris Cédex 13, France. Email: carole.dufouil@upmc.fr

First received 9 Feb 2010, final revision 10 Aug 2010, accepted 3 Nov 2010

\section{Funding}

The Three-City Study is conducted under a partnership agreement between the Institut National de la Santé et de la Recherche Médicale (INSERM), the Victor Segalen-Bordeaux II University, and Sanofi-Aventis. The Fondation pour la Recherche Médicale funded the preparation and initiation of the study. The $3 \mathrm{C}$ Study is also supported by the Caisse Nationale Maladie des Travailleurs Salariés, Direction Générale de la Santé, MGEN, Institut de la Longévité, Conseils Régionaux of Aquitaine and Bourgogne, Fondation de France, and de la Longevite, Conseils Regionaux of Aquitaine and Bourgogne, Fondation de France, and
Ministry of Research-INSERM Programme 'Cohortes et collections de données biologiques'. R.S. acknowledges funding from the National Institute for Health Research (NIHR) Specialist Biomedical Research Centre for Mental Health award to the South London and Maudsley NHS Foundation Trust and the Institute of Psychiatry, King's College London.

\section{References}

1 Ritchie K, Touchon J. Mild cognitive impairment: conceptual basis and current nosological status. Lancet 2000; 355: 225-8.

2 Geerlings $\mathrm{MI}$, Jonker $\mathrm{C}$, Bouter LM, Adèr HJ, Schmand B. Association between memory complaints and incident Alzheimer's disease in elderly people with normal baseline cognition. Am J Psychiatry 1999; 156: 531-7.

3 Kim J-M, Stewart R, Kim S-W, Yang S-J, Shin I-S, Yoon J-S. A prospective study of change in subjective memory complaints and onset of dementia in South Korea. Am J Geriatr Psychiatry 2006; 14: 949-56.

4 Schmand B, Jonker C, Geerlings MI, Lindeboom J. Subjective memory complaints in the elderly: depressive symptoms and future dementia. Br J Psychiatry 1997; 171: 373-6.

5 Palmer K, Bäckman L, Winblad B, Fratiglioni L. Detection of Alzheimer's disease and dementia in the preclinical phase: population based cohort study. BM J 2003; 326: 245-7.

6 Jorm AF, Christensen H, Korten AE, Henderson AS, Jacomb PA, Mackinnon A. Do cognitive complaints either predict future cognitive decline or reflect past cognitive decline? A longitudinal study of an elderly community sample. Psychol Med 1997; 27: 91-8.

7 Jonker C, Launer L, Hooijer C, Lindeboom J. Memory complaints and memory impairment in older individuals. J Am Geriatr SoC 1996; 44: 44-9.

8 Dufouil C, Fuhrer R, Alperovitch A. Subjective cognitive complaints and cognitive decline: consequence or predictor? The epidemiology of vascular aging study. J Am Geriatr SOC 2005; 53: 616-21.

9 Stewart R, Russ C, Richards M, Brayne C, Lovestone S, Mann A. Depression, APOE genotype and subjective memory impairment: a cross-sectional study in an African Caribbean population. Psychol Med 2001; 31: 431-40. 
10 Small GW, Chen ST, Komo S, Ercoli L, Bookheimer S, Miller K, et al. Memory self-appraisal in middle-aged and older adults with the apolipoprotein E-4 allele. Am J Psychiatry 1999; 156: 1035-8.

11 Barnes LL, Schneider JA, Boyle PA, Bienias JL, Bennett DA. Memory complaints are related to Alzheimer disease pathology in older persons. Neurology 2006; 67: 1581-5.

12 de Groot JC, de Leeuw F-E, Oudkerk M, Hofman A, Jolles J, Breteler MMB. Cerebral white matter lesions and subjective cognitive dysfunction. The Rotterdam Scan Study. Neurology 2001; 56: 1539-45.

13 Minett TS, Dean JL, Firbank M, English P, O'Brien JT. Subjective memory complaints, white-matter lesions, depressive symptoms, and cognition in elderly patients. Am J Geriatr Psychiatry 2005; 13: 665-71.

14 Stewart R, Dufouil C, Godin O, Ritchie K, Maillard P, Delcroix N, et al. Neuroimaging correlates of subjective memory deficits in a community population. Neurology 2008; 70: 1601-7.

15 Wang PJ, Saykin AJ, Flashman LA, Wishart HA, Rabin LA, Santulli RB, et al. Regionally specific atrophy of the corpus callosum in $A D, M C l$ and cognitive complaints. Neurobiol Aging 2006; 27: 1613-7.

16 Saykin AJ, Wishart HA, Rabin LA, Santulli RB, Flashman LA, West JD, et al. Older adults with cognitive complaints show brain atrophy similar to that of amnestic MCl. Neurology 2006; 67: 834-42.

17 van Norden AGW, Fick WF, de Laat KF, van Uden IWN, van Oudheusden LJB, Tendolkar I, et al. Subjective cognitive failures and hippocampal volume in elderly with white matter lesions. Neurology 2008; 71: 1152-1159.

18 3C Study Group. Vascular factors and risk of dementia: design of the Three-City Study and baseline characteristics of the study population. Neuroepidemiology 2003; 22: 316-25.

19 Godin O, Dufouil C, Maillard P, Delcroix N, Mazoyer B, Crivello F, et al. White matter lesions as a predictor of depression in the elderly: the 3C-Dijon study. Biol Psychiatry 2008; 63: 663-9.

20 Maillard P, Delcroix N, Crivello F, Dufouil C, Gicquel S, Joliot M, et al. An automated procedure for the assessment of white matter hyperintensities by multispectral (T1, T2, PD) MRI and an evaluation of its between-centre reproducibility based on two large community databases. Neuroradiology 2008; 50: 31-42.

21 Maillard P, Crivello F, Dufouil C, Tzourio-Mazoyer N, Tzourio C, Mazoyer B. Longitudinal follow-up of individual white matter hyperintensities in a large cohort of elderly. Neuroradiology 2009; 51: 209-20.
22 Gagnon M, Dartigues JF, Mazaux JM, Dequae L, Letenneur L, Giroire JM, et al. Self-reported memory complaints and memory performance in elderly French community residents: results of the PAQUID research program. Neuroepidemiology 1996; 13: 145-54.

23 Kim J-M, Stewart R, Shin I-S, Choi S-K, Yoon J-S. Subjective memory impairment, cognitive function and depression - a community study in older Koreans. Dement Geriatr Cogn Disord 2003; 15: 218-25.

24 Fuhrer $\mathrm{R}$, Rouillon $\mathrm{F}$. La version française de l'échelle CES-D [French version of the CES-D]. Psychiatrie Psychobiologie 1989; 4: 163-166.

25 American Psychiatric Association. Diagnostic and Statistical Manual of Mental Disorders (4th edn) (DSM-IV). APA, 1994.

26 Artero S, Ancelin M-L, Portet F, Dupuy A, Berr C, Dartigues JF, et al. Risk profiles for mild cognitive impairment and progression to dementia are gender specific. J Neurol Neurosurg Psychiatry 2008; 79: 979-84.

27 Folstein M, Folstein S, McHugh P. 'Mini-Mental State', a practical method for grading the cognitive state of patients for the clinician. J Psychiatr Res 1975; 12: $189-98$.

28 Isaacs B, Kennie AT. The Set Test as an aid to the detection of dementia in old people. Br J Psychiatry 1973; 45: 957-62.

29 Benton AL. Manuel pour L'application du Test de Rétention Visuelle. Applications cliniques et expérimentales [Manual for the Use of the Visual Retention Test. Clinical and Experimental Applications]. Centre de Psychologie Appliquée, 1965

30 Bolla KI, Lindgren KN, Bonaccorsy C, Bleecker ML. Memory complaints in older adults. Fact or fiction? Arch Neurol 1991; 48: 61-4.

31 Trouton $A$, Stewart $R$, Prince $M$. Does social activity influence the accuracy of subjective memory deficit? Findings from a British community survey. J Am Geriatr Soc 2006; 54: 1108-13.

32 Jorm AF, Butterworth P, Anstley KJ, Christensen H, Easteal S, Maller J, et al. Memory complaints in a community sample aged 60-64 years: associations with cognitive functioning, psychiatric symptoms, medical conditions, APOE genotype, hippocampus and amygdala volumes, and white-matter hyperintensities. Psychol Med 2004; 34: 1495-506.

33 Kim KW, MacFall JR, Payne ME. Classification of white matter lesions on magnetic resonance imaging in elderly persons. Biol Psychiatry 2008; 64 273-80.

\section{poems by
doctors}

\section{Intimacy}

Roy Salole

Across the road we stand, strangers

the comfortable distance reassuring enough

to let us wave and smile.

But when we meet

in the heat of confrontation

face to face and stripped,

we break into sweat, like in a sauna,

and our tongues are parched and speechless.

This is the moment to embrace nakedly and unashamed, but mostly we turn our backs and walk away.

Other poems by Salole have been published in the December 2009 and September 2010 issues of the Journal. 